\title{
Fetal Congenital Peripheral Bronchial Atresia Diagnosed by Magnetic Resonance Imaging: Two Case Reports
}

\author{
Mitsuru Kozaki, MD ${ }^{1}$ Yuko Iraha, MD, PhD ${ }^{2}$ Hitoshi Masamoto, MD, PhD ${ }^{1}$ Hayase Nitta, MD ${ }^{1}$ \\ Yukiko Chinen, $\mathrm{MD}^{1}$ Tadatsugu Kinjo, MD ${ }^{1}$ Keiko Mekaru, MD, PhD ${ }^{1}$ Yoichi Aoki, MD, PhD ${ }^{1}$ \\ ${ }^{1}$ Department of Obstetrics and Gynecology, Graduate School of \\ Medicine, University of the Ryukyus, Okinawa, Japan \\ 2 Department of Radiology, Graduate School of Medicine, University \\ of the Ryukyus, Okinawa, Japan \begin{abstract}
Obstetrics and Gynecology, Graduate School of Medicine, University of the Ryukyus, 207 Uehara Nishihara, Okinawa 903-0215, Japan (e-mail: yoichi@med.u-ryukyu.ac.jp).
\end{abstract} \\ Address for correspondence Yoichi Aoki, MD, PhD, Department of
}

Am J Perinatol Rep 2018;8:e201-e205.

\begin{abstract}
Keywords

- congenital bronchial atresia

- prenatal diagnosis

- magnetic resonance imaging

- ultrasonography

Two types of congenital bronchial atresia (proximal and peripheral) have been classified. We report two cases of peripheral bronchial atresia diagnosed by prenatal ultrasonography (US) and magnetic resonance imaging (MRI). Evaluating an enlarged lung mass that is homogeneously hyperechoic on US and hyperintense on T2-weighted MRI can help in determining whether bronchial atresia is present. Proximal type is suggested when a dilated main bronchus is observed as a tubule structure of an involved lung hilum. In our cases, T2-weighted MRI revealed homogeneously hyperintense lung lesion with decreased signal intensity of adjacent lobe, flattening diaphragm, and mediastinal shift. Dilatation of the main bronchus was not observed and the opposite lung was normal in appearance. These findings were explained by secondary compression due to enlargement of the involved lung. The preservation of vascular structure and the retained normal shape, though enlarged, in the affected lobe were observed, which demonstrated undisrupted pulmonary architecture of the lobe. Thus, congenital cystic adenomatoid malformation was excluded because pulmonary architecture was relatively preserved. Finally, presumed diagnoses of the peripheral bronchial atresia were made and confirmed by postnatal chest computed tomography.
\end{abstract}

Bronchial atresia is a rare disease of uncertain etiology that can occur anywhere along the bronchial tract. Prenatally, the airway distal to the obstruction becomes dilated and fluid-filled. Segmental obstruction affects only a small volume of the lung and usually has almost no clinical consequence. It is usually discovered by chance or with recurrent respiratory infections in neonate, later in childhood, or adulthood. Obstruction of the mainstem or the proximal lobar bronchi, however, can lead to a greater loss of normal lung volume, resulting in severe, often lethal consequences. $^{1-3}$

received

April 25, 2018

accepted after revision

August 21, 2018
DOI https://doi.org/

10.1055/s-0038-1673620. ISSN 2157-6998.
The bronchial atresia is characterized by emphysematous change of one or more of the pulmonary lobes. Routine prenatal ultrasonography (US) has resulted in early detection, which can lead to serial evaluation. Here, we report two cases diagnosed as fetal peripheral bronchial atresia by prenatal US and magnetic resonance imaging (MRI).

\section{Case Reports}

Case 1: A 38-year-old woman, para 3-0-0-3, was identified with fetal abnormalities, involving a leftward shift of the
Copyright $\odot 2018$ by Thieme Medical Publishers, Inc., 333 Seventh Avenue, New York, NY 10001, USA. Tel: +1(212) 584-4662.
License terms

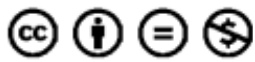




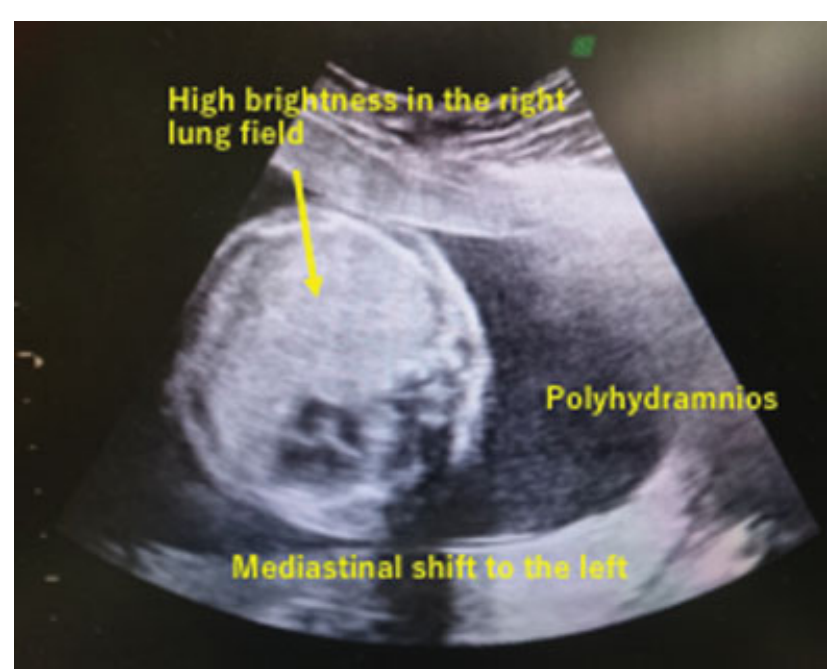

Fig. 1 Fetal ultrasonography at 26 weeks of gestation shows a leftward shift of the mediastinum, hyperechoic lesion in the right lung field, and polyhydramnios.

mediastinum, hyperechoic lesion in the right lung field, and polyhydramnios by US at 26 weeks of gestation (- Fig. 1). Thereafter, serial US follow-up was performed and polyhydramnios was improved gradually. T2-weighted MRI at 31 weeks of gestation showed high signal intensity of the enlarged mid and lower lobes, and low signal intensity of the upper lobe of the right lung with flattening diaphragm. Although mediastinal shift to the left side and slight compression of the left lung were observed, the left lung was almost normal in appearance. Furthermore, the preservation of vascular structure in the right mid and lower lobes and the retained normal shape of the lobes demonstrated undisrupted pulmonary architecture of the lobes (-Fig. 2). These findings lead to the presumed diagnosis of fetal congenital right mid and lower lobar bronchial atresia. Thereafter, her pregnancy was uneventful. At $38^{2 / 7}$ weeks of gestation, a 2,578-g boy infant was delivered with Apgar scores 8/8 through a repeated cesarean section. The baby required oxygen treatment for 4 months. Chest computed tomography (CT) at 3 months of age showed emphysematous changes in right S4 and S6 regions and expanded bronchus filled with secretions (-Fig. 3). Peripheral bronchial atresia was confirmed. The baby required oxygen treatment for 4 months and his respiratory state was uneventful thereafter. However, he underwent the right mid and lower lobectomy due to the upper lobe compression at 2 years and 7 months of age. No treatment was necessary after that and he is healthy now at 4 years and 4 months of age.

Case 2: At 30 weeks of gestation, a 33-year-old woman, para 1-0-0-1, was identified with fetal abnormalities involving hyperechoic lesion in the left lung field with microcystic pattern and a mediastinal right-side shift by US ( - Fig. 4). She was referred to our hospital, because a microcystic type of congenital cystic adenomatoid malformation (CCAM) was suspected. T2-weighted MRI at 31 weeks of gestation showed volume increase, and high signal intensity of the upper lobe and low signal intensity of the lower lobe of the left lung with flattening diaphragm. Although mediastinal shift to the right side was observed, the right lung was normal in appearance. The fetal dilated main bronchus and microcystic structure could not be found, but the fetal trachea and both main bronchi were depicted. Furthermore, the preservation of vascular structure in the left upper lobe and the retained normal shape of the lobe demonstrated undisrupted pulmonary architecture of the lobe. Trachea and bilateral bronchi were depicted (-Fig. 5). These findings lead to the presumed diagnosis of fetal congenital bronchial atresia of the left upper lobe. At that time, we missed a trapezoid lesion with extremely high signal intensity in the left lower lobe
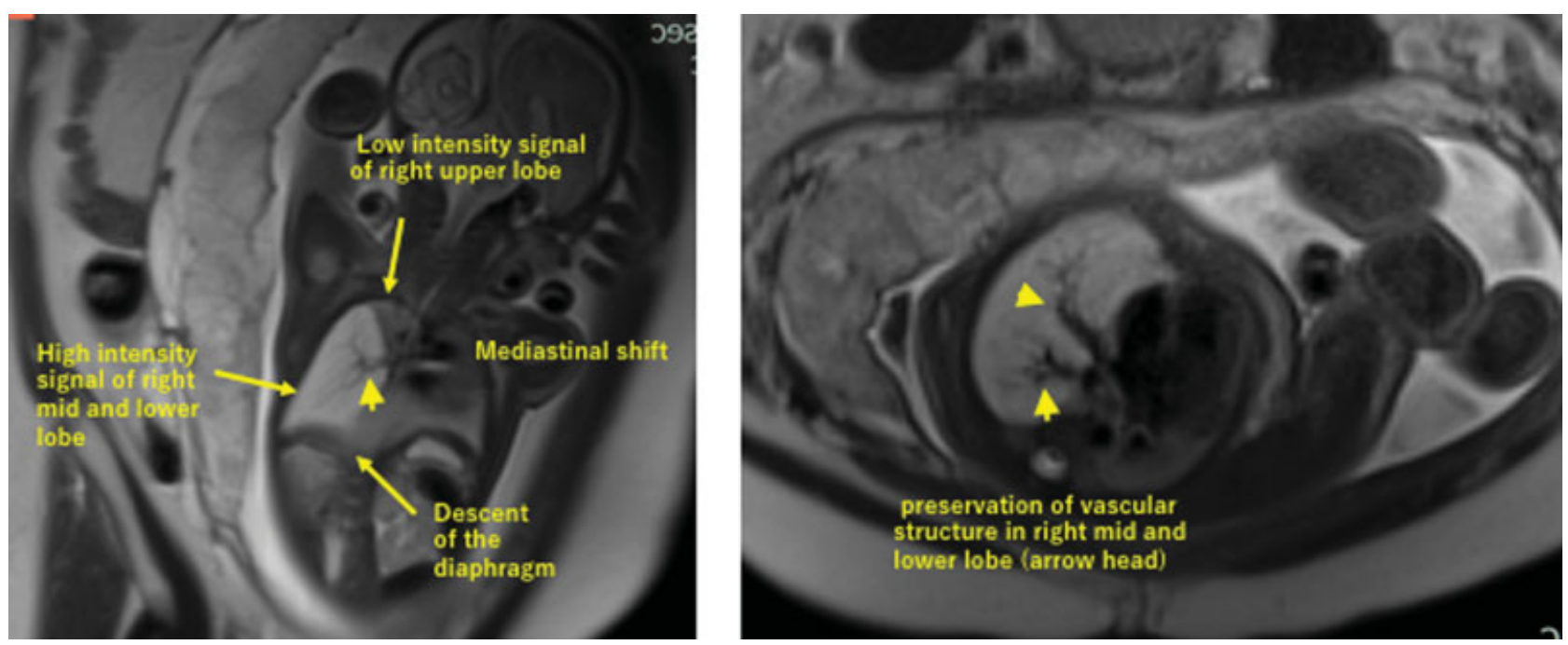

Fig. 2 T2-weighted magnetic resonance imaging (HASTE [half-Fourier single-shot turbo spin-echo]) at 31 weeks of gestation shows high signal intensity of the enlarged mid and lower lobes of the right lung with compression of the right upper lobe, mediastinal shift to the left side, and flattening diaphragm. Although mediastinal shift to the left side and slight compression of the left lung were observed, the left lung was almost normal in appearance. Furthermore, the preservation of vascular structure in the right mid and lower lobes (arrowheads) and the retained normal shape of the lobes are depicted. 


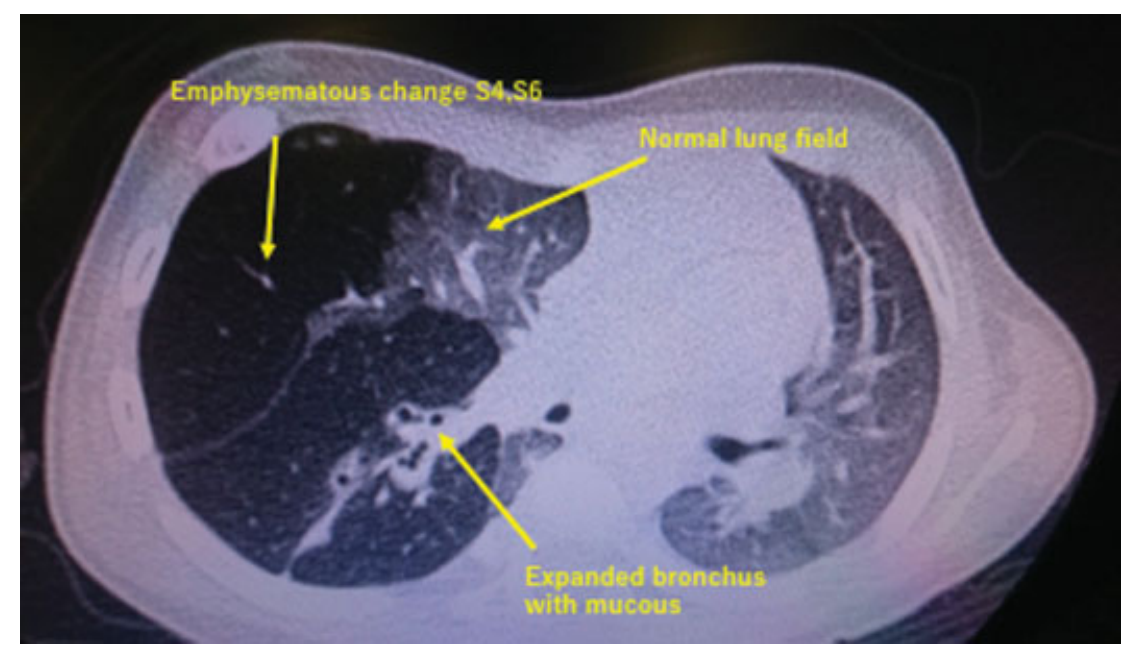

Fig. 3 Chest computed tomography at 3 months of age shows emphysematous changes in right S4 and S6 regions and expanded bronchus filled with secretions.

(-Fig. 5, asterisks). This lesion was found to be the left extralobar pulmonary sequestration, which was diagnosed by chest CT at 2 years after birth. Thereafter, her pregnancy was uneventful. At 40 weeks of gestation, a 2,920-g boy infant with Apgar score 8/8 was vaginally delivered. The infant's respiratory status was stable. Transverse chest CT at 1 year showed emphysematous changes in the left upper lobe and expanded bronchus filled with secretions (-Fig. 6). The peripheral bronchial atresia in the left upper lobe was confirmed. Finally, the left extralobar pulmonary sequestration was diagnosed by chest CT at 2 years of age and no treatment has been required. He is healthy now at 9 years and 3 months of age.

\section{Discussion}

The prenatal imaging findings for CCAM, bronchopulmonary sequestrations, and bronchogenic cysts have been typically demonstrated in the previous reports and a prenatal diagnosis

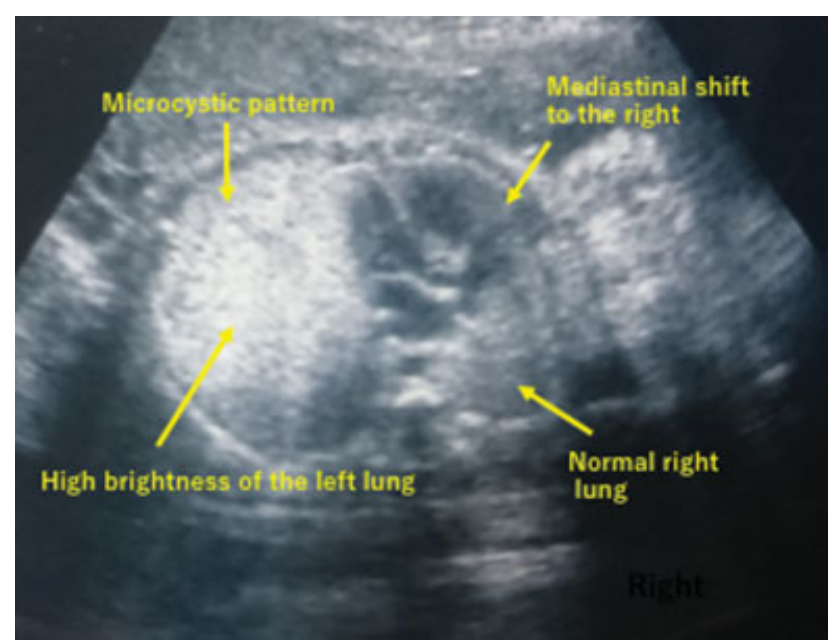

Fig. 4 Fetal ultrasonography at 30 weeks of gestation shows hyperechoic lesion in the left lung field with microcystic pattern and a mediastinal right-side shift. of these abnormalities is made precisely. ${ }^{4,5}$ On the other hand, bronchial atresia is a rare disease and reveals a focal disappearance of a bronchus with normal architecture of the distal lung. The airway obstruction leads to the accumulation of the distally produced pulmonary fluid, revealing pulmonary expansion, homogeneously increased echogenicity, and high signal intensity on T2-weighted MRIs. These prenatal findings, however, are not specific, and were observed in other congenital lung malformations already described. ${ }^{6}$

By location, imaging, and clinical findings, two types of congenital bronchial atresia (proximal and peripheral) have been identified. ${ }^{7,8}$ The proximal bronchial atresia is located at the level of the mainstem or the proximal lobar bronchi. Although it is very rare, prenatal examinations often correctly identify the atresia, which causes a greater increase of the distal lung, with homogeneously hyperechogenic lesion by US and hyperintensity by T2-weighted MRI. Usually, eversion of the diaphragm, mediastinal shift, and severe compression of the normal lung are observed due to the pathologically large abnormal lung. A centrally located, fluid-filled, tubular structure (a bronchocele) is often depicted due to the dilated bronchus distal to the atresia. ${ }^{9}$ Cardiac failure due to the inferior vena cava compression and the pulmonary hypoplasia causes lethal consequences prenatally or neonatally. 7,8

The peripheral bronchial atresia is located at the segmental bronchial level and is often asymptomatic and managed without operation. However, peripheral bronchial atresia has been reported to be associated with other anomalies in a complex malformation, where most of them are the microcystic type of CCAMs. It was demonstrated that $65 \%$ of peripheral bronchial atresia initially resided in microcystic CCAMs. ${ }^{10}$ The prenatal diagnosis of the bronchial atresia in these cases seems to be difficult because typical imaging findings that may hide the bronchial atresia were observed in the associated malformations. ${ }^{5,11}$

Evaluating an enlarged lung mass which is homogeneously hyperechoic on US and hyperintense on T2-weighted MRI can help in determining whether bronchial atresia is present. Proximal type is suggested when a dilated main bronchus is 

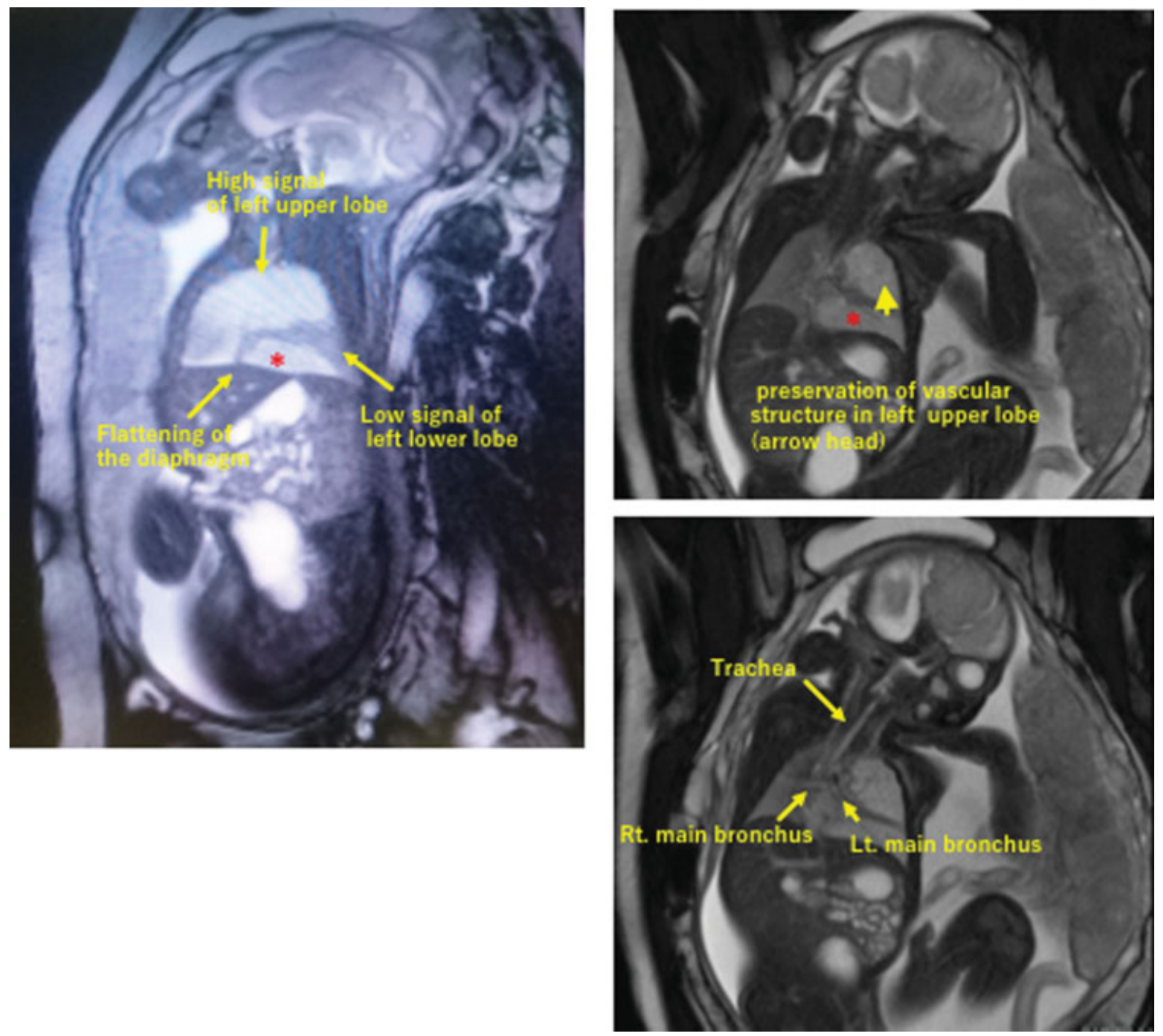

Fig. 5 Sagittal T2-weighted magnetic resonance imaging (true-FISP [steady-state free precession imaging]) at 31 weeks of gestation shows high signal intensity of the enlarged upper lobe and compression of the lower lobe of the left lung with flattening diaphragm. The fetal trachea and both main bronchi are depicted. Furthermore, the preservation of vascular structure in the left upper lobe (arrowhead) and the retained normal shape of the lobe are observed. Trachea and bilateral bronchi were depicted. Axial and sagittal views revealed a mediastinal shift to the right side (not shown). Asterisk indicates a trapezoid lesion with extremely high signal intensity in the left lower lobe.

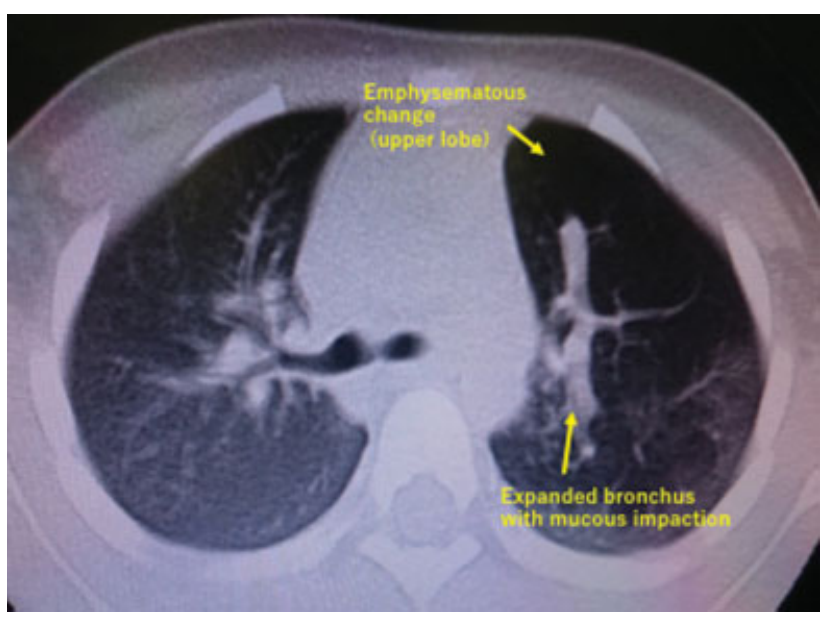

Fig. 6 Transverse chest computed tomography at 1 year shows emphysematous changes in the left upper lobe and expanded bronchus filled with secretions. observed as a tubule structure of an involved lung hilum, which might be better detected by fetal MRI. ${ }^{8}$ In our cases, T2weighted MRI revealed homogeneously hyperintense lung lesion with decreased signal intensity of adjacent lobe, flattening diaphragm, and mediastinal shift to the contralateral side. Unlike the proximal bronchial atresia, dilatation of the main bronchus was not observed, and the opposite lung was normal in appearance. Decreased signal intensity of the adjacent lobe, flattening diaphragm, and mediastinal shift were explained by secondary compression due to enlargement of the involved lung. It may be possible to diagnose the peripheral type of the bronchial atresia prenatally if the preservation of vascular structure and the retained normal shape, though enlarged, in the affected lobe are observed, which demonstrate undisrupted pulmonary architecture of the lobe. Furthermore, CCAM was excluded because pulmonary architecture was relatively preserved. Finally, presumed diagnoses of the 
peripheral bronchial atresia were made and confirmed by postnatal chest CT.

It is very important to differentiate between peripheral and mainstem bronchial atresia. The mainstem atresia is of very poor prognosis, because cardiac failure due to the inferior vena cava compression and the pulmonary hypoplasia causes lethal consequences prenatally or neonatally. 7,8 Also, when detecting peripheral bronchial atresia, a complex lung malformation should be considered, particularly the microcystic type of CCAM.

\section{Conflict of Interest}

The authors declare that there is no conflict of interest regarding the publication of this paper.

\section{Acknowledgments}

The authors would like to thank Enago (www.enago.jp) for the English language review of this article.

\section{References}

1 Berrocal T, Madrid C, Novo S, Gutiérrez J, Arjonilla A, Gómez-León $\mathrm{N}$. Congenital anomalies of the tracheobronchial tree, lung, and mediastinum: embryology, radiology, and pathology. Radiographics 2004;24(01):e17
2 Langston C. New concepts in the pathology of congenital lung malformations. Semin Pediatr Surg 2003;12(01):17-37

3 Kunisaki SM, Fauza DO, Nemes LP, et al. Bronchial atresia: the hidden pathology within a spectrum of prenatally diagnosed lung masses. J Pediatr Surg 2006;41(01):61-65, discussion 61-65

4 Alamo L, Gudinchet F, Reinberg O, et al. Prenatal diagnosis of congenital lung malformations. Pediatr Radiol 2012;42(03):273-283

5 Cannie M, Jani J, De Keyzer F, et al. Magnetic resonance imaging of the fetal lung: a pictorial essay. Eur Radiol 2008;18(07):1364-1374

6 Alamo L, Reinberg O, Vial Y, Gudinchet F, Meuli R. Comparison of foetal US and MRI in the characterisation of congenital lung anomalies. Eur J Radiol 2013;82(12):e860-e866

7 Zamora IJ, Sheikh F, Olutoye OO, et al. Mainstem bronchial atresia: a lethal anomaly amenable to fetal surgical treatment. J Pediatr Surg 2014;49(05):706-711

8 Abitayeh G, Ruano R, Martinovic J, Barthe B, Aubry MC, Benachi A. Prenatal diagnosis of main stem bronchial atresia using 3-dimensional ultrasonographic technologies. J Ultrasound Med 2010;29 (04):633-638

9 Alamo L, Vial Y, Gengler C, Meuli R. Imaging findings of bronchial atresia in fetuses, neonates and infants. Pediatr Radiol 2016;46 (03):383-390

10 Peranteau WH, Merchant AM, Hedrick HL, et al. Prenatal course and postnatal management of peripheral bronchial atresia: association with congenital cystic adenomatoid malformation of the lung. Fetal Diagn Ther 2008;24(03):190-196

11 Epelman M, Kreiger PA, Servaes S, Victoria T, Hellinger JC. Current imaging of prenatally diagnosed congenital lung lesions. Semin Ultrasound CT MR 2010;31(02):141-157 